\title{
XIX. \\ Ein Beitrag zur Aetiologie der multiplen Neuritis in den Tropen.
}

\author{
Von Dr. Max Glogner, \\ prakt. Arzt in Samarang.
}

Als ich am Ende des Jahres 1892 meine Untersuchungen über den klinischen Verlauf und die Ursache der Beri-Berikrankheit begann, wählte ich mir für die Bearbeitung des zweiten, ätiologischen Theiles absichtlich Fälle aus, welche bereits längere Zeit im Krankenhause verpflegt wurden und bei denen die klinischen Erscheinungen in besonders hochgradigem Maasse zu Tage traten, weil ich vermuthen durfte, dass gerade bei ihnen die Krankheitserreger in reichlicher Anzahl vorhanden sein würden. Es waren dies Fälle, von denen der grösste Theil an leichten intermittirenden Fiebern litt, eine Erscheinung, welche nach der Erfahrung der meisten Autoren bei Beri-Berikranken häufig beobachtet wird und z. B. von Scheube und Baelz in je 45 und 50 pCt. der beobachteten Fälle gefunden wurde. Wie ich bereits in einer kurzen Bemerkung auf S. 60 des 132. Bandes dieses Archivs anführte, fand ich in den rothen Blutkörperchen in den meisten Fällen unter einigen 20 Beobachtungen Organismen in lebendem Zustande, welche ich ihrer Form und Beschaffenheit nach zu den Amöben rechnete. Obwohl diese Organismen bezüglich der Farbe und Form ihres Zellenleibes, sowie der Bewegung kleiner Pigmentkörnchen mit den von Laveran und Anderen gefundenen und mir von früher untersuchten Malariafällen bekannten Plasmodien die grösste Aehnlichkeit besassen, so glaubte ich doch, dass ich es hier mit einer von den Malariaplasmodien biologisch verschiedenen Art zu thun hätte, weil die sensiblen und motorischen Störungen an den unteren Extremitäten und die Herzerscheinungen während und direct nach den Fieberanfällen eine unverkennbare Zunahme erkennen liessen. Sehen wir ja auch bei den Bakterien zwei Arten von derselben oder ähnlichen 
Form in ihrem schädlichen Einflusse auf den menschlichen Organismus sich wesentlich unterscheiden. So dachte ich mir, dass die in rothen Blutzellen gefundenen Organismen trotz ihrer Aehnlichkeit mit den Plasmodien der Malaria eine besondere Art darstellen möchten. Ich wurde in dieser Anschaunng bestärkt, nachdem ich im Besitze der elektrischen Apparate die $\mathrm{Zu}$ - und Abnahme der Erscheinungen während und nach den Fieberanfällen genauer feststellen konnte.

Bei einer Reihe weiterer Beobachtungen, bei denen unter Temperaturerhöhung die Erscheinungen einer multiplen Neuritis auftraten, wurden nun nicht nur diese endoglobulären, amöboiden, sondern auch die von Laveran entdeckten pigmentirten, sichelförmigen und ovalen Gebilde gefunden. Der negative Befund bei einer grossen Anzahl von multiplen Neuritiden, welche ebenfalls mit Temperaturerhöhungen verliefen, liessen mich immer mehr an der Bedeutung dieser endoglobulären Organismen als Erreger der Beri-Berikrankheit zweifeln, und brachten mir die Ueberzeugung, dass ich es hier mit Malariaerkrankungen zu thun hatte, welche eine multiple Neuritis im Gefolge hatten. Aus Europa liegt eine grosse Anzahl von Beobachtungen vor, wo durch eine Infectionskrankheit, wie Diphtheritis, Typhus, Influenza u. s. w., eine multiple Neuritis entstand.

Die folgenden Beobachtungen sollen den Beweis erbringen, dass auch Malariaerkrankungen derartige Affectionen, die in Gegenden, wo Beri-Beri vorkommt, meist als Beri-Bori bezeichnet werden, hervorrufen können.

Der 25 Jahre alte, gut gebaute, javanische Sträfling S. wurde wegen einer Contusion am rechten Hüftgelenk im October 1892 in's Krankenhaus aufgenommen. Anfang December bekam er Schmerzen in beiden Unterschenkeln, im December und Januar litt er an leichten atypischen Fieberanfällen. Am 19. Februar trat ein heftiger Anfall auf: Temp. $40,3^{\circ} \mathrm{C}$., heftige, gesteigerte Schmerzen in beiden Unterextremitäten; am 28. Februar wiederholte sich das Fieber, Temp. $39,3^{\circ} \mathrm{O}$, Puls 116, Athmung 28 pro Min. Milz vergrössert. Vom 19:-28. Februar war der Puls, ohne Temperaturerhöhung, verschnellt und schwankte zwischen 90 und 112 Schlägen pro Min. Nach dem letzten Fieberanfall hatte der Kranke ausser intensiven Schmerzen und Ameisenlaufen noch ein Gefübl von Wärme in beiden unteren Extremităten. Die Nerven und Muskeln der unteren und oberen Extremitäten waren auf Druck schmerzhaft. Patellar- und Achillesreflexe nicht vorhanden. Herzschlag beschleunigt; keine Geräusche oder Vergrösserung. Am 7. März 
wurden beide Nervi peronei und tibiales, sowie die Musculi tibiales antici elektrisch untersucht. Ich wiederhole und bemerke, dass bei gesunden Eingebornen die Zablen fär die minimalste Contraction bei Kathodenschliessungszuckung an den Nervi peronei und tibiales an meinem Galvanometer zwischen $1 \frac{1}{2}$ und $7 \frac{1}{2}$ Milliampères, diejenigen für die Musculi tibiales antici bei directer Reizung $\mathbf{z w i s c h e n} 3$ und 8 Milliampères lagen und dass der Abstand der secundären von der primären Rolle bei dem mir zu Gebote stehenden Dubois-Reymond'schen Schlittenapparat an den betreffenden Nerven und Muskeln stets grösser als 35 gefunden wurde.

Das Resultat der elektrischen Untersuchung war bei unserem Kranken das folgende:

rechts

Musc. tib. antic.: galv. $<8 \frac{1}{2}$ Milliampères, farad. 35

$\begin{array}{ccrcc}\text { Nerv. peron.: } & - & 4 \frac{1}{2} & - & - \\ -\quad \text { tib.: } & - & 8 \frac{1}{2} & - & - \\ & & \text { links } & & \end{array}$

Musc. tib. antic.: galv. $6 \frac{1}{2}$ Milliampères, farad. 41

Nerv. peron.: $\quad-8 \frac{1}{4} \quad-\quad-23$

- tib.: - $9 \frac{1}{2} \quad-\quad-30$.

Es bestand demnach Ieichte Herabsetzung der galvanischen Reizbarkeit am Nerv. tibial, und Musc. tib. antic. rechts, sowie Herabsetzung der Reizbarkeit auf beide Stromarten an den Nerv. peron. und tibial. der linken Seite. Am 8. März kehrte das Fieber zurück, welches mit Ausnahme des 9. März bis zum 21. anbielt: Temp. $38,4^{\circ} \mathrm{C}$. Morgens, Puls 112, Athmung 24 pro Min. am 8. März; am 10. März Morgentemperatur $38,1^{\circ} \mathrm{C}$., Puls 108, Athmung 20; am 11. März $38^{\circ} \mathrm{C}$, Puls 102, Athmung 20 pro Min., Abends $39,2^{\circ} \mathrm{C}$; am 12. März 40,8 $8^{\circ}$ C. Morgens, Puls 116, Athmung 24. Vom 12. bis 21. März bestand ein unregelmässiges Fieber, welches zwischen $38^{\circ} \mathrm{C}$. und $39,5^{\circ} \mathrm{C}$. schwankte. Am 19. März wurde Folgendes notirt: Patient klagt über heftige Schmerzen und Formication in beiden unteren Extremitäten. Tast- und Ortsinn an beiden Unterschenkeln aufgehoben, ebenso das Schmerzgefühl und der Temperatursinn. Patient ist kurzatbmig, hat schlechten Appetit und Schlaf. Patellar- und Achillessehnenreflexe aufgehoben, Händedruek stark herabgesetzt, sensible Störungen an den oberen Extremitäten nicht wahrzunehmen. Dorsalflexion beider Füsse herabgesetzt, Gang sebr steif. Die Untersuebung mit dem elektrischen Strom ergab das folgende Resultat:

rechts

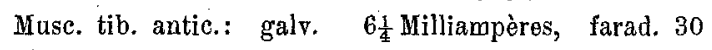

Nerv. peron.: $\quad-\quad 5 \frac{3}{4} \quad-\quad$ - 19

- tib.: - <11 -

links

Musc. tib. antic.: galv. 7 Milliampères, farad. 15, Contraction langsam Nerì. peron.:

- tib.:

-10
$-\quad 10$

$-\quad<10$
- nicht reizbar

-25 . 
In der Fieberzeit vom 8. bis 19. März war also eine deutliche Zunahme der elektrischen Abweichungen eingetreten. Vom 22. März bis 2. April hatte Patient kein Fieber. Schon am 31. März war eine bedeutende Besserung an den betreffenden Nerven und Muskeln zu constatiren; es wurden folgende Wertbe festgestellt:

rechts

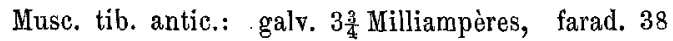

Nerv, peron.: $\quad-5 \frac{7}{2} \quad-\quad-37$

- tib.: $\quad-6 \frac{1}{2} \quad-\quad-35$

links

Musc. tib. antic.: galv. $6 \frac{1}{2}$ Milliampères, farad. 31

Nerv. peron.: $\quad-1 \frac{1}{2} \quad-\quad-36$

- tib.: $\quad-8$ - 8 - 36 .

Am 2., 10., 13. April noch leichte Fieberanfälle, nach deren Ablauf der Puls stets noch einige Tage verschnellt blieb. Am 2. Mai ist der Zustand wie folgt: Nur im rechten Unterschenkel fühlt der Kranke noch Schmerzen, in beiden Füssen bat er ein Gefühl von Wärme. In den oberen Extremitäten fühlt er nichts, Händedruck kräftig. Schmerz auf Druck bestebt nur noch in den Wadenmuskeln, im rechten Cruralis, sowie in den Muskeln der rechten oberen Extremität. Bewegung der Füsse frei, rechter Patellarreflex erhalten, der linke, sowie die Achillessehnenreflexe nicht; alle Empfindungsqualitäten überall erhalten. Der Zustand besserte sich allmählich; Pat. wurde geheilt entlassen. Am Abende des 11. März wurde sein Fingerblut untersucht; es fanden sich durcbschnittlich in jedem Präparat 3 endoglobuläre Amöben. Ihr Plasma war weisslich, mit deutlicher Bewegung der Pigmentkörnchen; ihre Grösse schwankte zwischen $\frac{1}{4}-\frac{1}{2}$ der Grösse eines rothen Blutkörperchens. Bei den grösseren bewegten sich die Pigmentkörnchen in der Peripherie der unregelmässig geformten Zellenleiber. Die rothen Blutzellen waren abgeblasst, jedoch nicht vergrössert. Es war vorauszusehen, dass nach der Sporulation der grösseren ein erhöhtes Fieber sich einstellen würde. Dasselbe trat am folgenden Morgen ein: Temp. 40,8 $\mathrm{C}$, Puls 116 , Athmung 24. Es war damit sichergestellt, dass das Fieber, an dem Patient litt, mit der Entwickelung der erwähnten Organismen in Zusammenhang stand. Die klinische Beobachtung ergab eine Zunahme der Erscheinungen an Nerven und Muskeln der Unterschenkel während des Fiebers und eine Abnahme derselben nach Ablauf desselben.

Fall 2.

Der Chinese G. T. Tj. wurde am 21. September 1894 mit den Erscheinungen der Beri-Berikrankheit in's Krankenhaus aufgenommen. Er war früher stets gesund gewesen. Seit 18 Tagen war er krank, die ersten 10 Tage litt er an Fieber, welches unter Schüttelfrost jeden Morgen ungefäbr um 8 Uhr anfing, den ganzen Tag anbielt und am Abend endete; während der Nacht schwitzte er stark. In der Fieberzeit fühlte er stets Müdigkeit. Schwäche in den Unterschenkeln, sowie Ameisenlaufen in den oberen uno 
unteren Extremitäten. Seit 8 Tagen, direct nach Ablauf des Fiebers, bekam er Oedeme der Füsse und Untersehenkel. Jetzt füblte er Ameisenlaufen in den oberen und unteren Extremitäten und Schwere in den unteren, er kann aus der hockenden Stellung sich nicht allein erheben. Händedruck beiderseits stark herabgesetzt. Nirgends Druckschmerz. Starkes Oedem der Füsse und Unterschenkel. Alle Empfindungsqualitäten erbalten; ebenso alle Reflexe, mit Ausnahme beider Patellar- und Achillessehnenreflexe. Dorsalflexion der Füsse herabgesetzt; Herz nicht vergrössert, überall systolische, blasende Geräusche; sichtbare Schleimhäute stark anämisch, Hämoglobin 75 pCt. Milz vergrössert. Patient batte vom 22.-30. September normale Pulszahl, Athmung und Temperatur. Am 21. September wurde sein Blut untersucht und eine grosse Anzahl der sichelförmigen und extraglobulären, amöboiden, pigmentirten Gebilde gefunden; an demselben Tage wurden die Nervi peronei und tibiales, sowie die Muse. tibial. antic. elektrisch untersucht und folgende Werthe festgestellt:

$$
\text { rechts }
$$

Musc. tib. antic.: galv. < 10 Milliampères, farad. 31

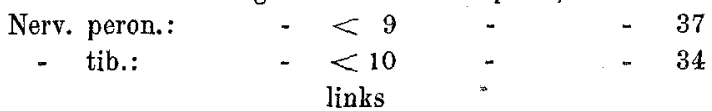

Musc. tib. antic.: galv. < 10 Milliampères, farad. 34

Nerv. peron.: $\quad-\quad 9 \frac{1}{2} \quad-\quad-28$

- tib.: - $<10 \quad-\quad 41$.

Es bestand demnach Herabsetzung der galranischen Reizbarkeit an allen Nerven und Muskeln und Herabsetzung der Reizbarkeit auf den faradischen Strom in leichtem Grade am rechten Musc. tib. antic. und linken Nerv. peroneus. Am 30. September bekam er ein leichtes Fieber: $38^{\circ} \mathrm{C}$, Puls 106, Respiration 24; am 2. October ein Fieberanfall von $39,9^{\circ} \mathrm{O}$., Puls 104, Athmung 24. Von diesem Tage an blieben Temperatur, Puls und Athmung bis zu seiner Entlassung am 13. October normal. Er füblte an diesem Tage bei der Untersuchung weder Schwäche, noch Ameisenlaufen. Händedruck rechts kräftig, links leicht herabgesetzt. Bewegungen der Füsse frei. Die Werthe für die elektrische Reizbarkeit waren die folgenden:

rechits

Musc. tib. antic.: galv. 5 Milliampères, farad. 48

Nerv. peron.: $\quad-5 \frac{1}{2} \quad-\quad-45$

- tib.: $\quad-6 \frac{1}{4} \quad-\quad-41$

links

Musc. tib. antic.: galv. $6 \frac{1}{2}$ Milliampères; farad. 48

Nerv. peron.: $\quad-8 \quad-\quad-48$

- tib.: - $6 \frac{1}{2}-47$ -

Patient wurde von seiner Aufnahme an mit grossen Dosen Chinin behandelt, mit dem Resultate, dass am 13. October die elektrischen Abweichungen verschwunden waren. Als an diesem Tage sein Blut untersucht wurde, fand ich in derselben Anzahl von Präparaten kein einziges Plasmodium, 
Fall 3.

Der Gefangene Sam. wurde Ende Januar 1894 in's Krankenhaus aufgenommen. Er fühlte sich seit 6 Tagen unwohl, hatte Ameisenlaufen in den unteren und oberen Extremitäten und eine auffallende Schwäche in Armen und Beinen. Ueber diese letzten Erscheinungen klagte er noch bei seiner Aufnahme, er läuft steif, Druckschmerz in den Muskeln und Nervenstämmen der oberen und unteren Extremitäten, alle Empfindungsqualitäten erhalten. Patellar - und Achillessehnenreflexe erloschen. Dorsalflexion beider Füsse stark herabgesetzt. Herzarbeit beschleunigt. Puls.90 pro Min.; Temp. 36,80 O. Die Untersuchung auf beide Stromarten ergab folgende Resultate:

rechts

Musc. tib. antic.: galv. $5 \frac{1}{2}$ Milliampères, farad. 45

Nerv, peron.: $\quad-4 \frac{1}{4} \quad-\quad-48$

- tib.: $\quad-2 \frac{1}{2} \quad-\quad-41$

links

Musc. tib. antic.: galv. $7 \frac{1}{2}$ Milliampères, farad. 40

Nerv. peron.: $\quad-5 \frac{1}{2} \quad-\quad-47$

- tib.: - $5 \frac{1}{2}$ - $\quad 40$.

Es wurden demnach keine elektrischen Abweichungen gefunden. Bis zum 2. Februar war die Zahl der Pulsschläge bei normaler Temperatur und Athmung vermehrt, vom 2.-10. Februar war auch der Puls in normale Grenzen zurückgekebrt. Am 10. Februar wurde ein Fieberanfall beobachtet: Temp. $39,5^{\circ}$ C.; am 12. Februar wiederholte sich das Fieber: Temp. 40,10 C.; am 14. Februar ein neuer Anfall, $38,3^{\circ} \mathrm{O}$., Milz vergrössert, Puls 96, Athmung 24 pro Min. Am 14. Februar wurden folgende elektrische Werthe gefunden:

rechts

Musc. tib. antic.: galv. 4 Milliampères, farad. 35, Contraction langsam Nerv, peron.: $\quad-\quad 2 \quad$ - $\quad 2 \quad-37$

- tib.: $\quad-\quad<7 \quad-\quad-41$

links

Musc. tib. antic.: galv. 4 Milliampères, farad. 32

Nerv. peron.: $\quad-2 \frac{1}{3} \quad-\quad-35$

- tib.: - $5 \frac{1}{2} \quad-\quad 39$.

Die Werthe für die Reizbarkeit auf den faradischen Strom näherten sich am Musc. tib. antic. und Nerv. peron. links bereits dem abnormen Gebiet. Vom 14.-25. Februar blieb der Puls bei normaler Temperatur und Athunng beschleunigt. Am 22. Februar klagte Patient über heftige Schmerzen und Formication in den unteren Extremitäten, so dass er nicht im Stande war, zu laufen. Temperatursinn war an der rechten Fusssoble aufgeboben, alle anderen Empfindungsqualitäten normal. Appetit schlecht, Schlaf der Schmerzen wegen gestört. Blasen- und Mastdarmfunctionen normal. Am 25. Februar begann eine Reihe von neven Fieberanfällen: 
Temperatur Puls Athroung

Am 25. Februar Morgens $36,9^{\circ}$ C. A A bends $38,6^{\circ}$ C. $104 \quad 24$

$\begin{array}{llllllll}-26 . & - & - & 38,1 & - & - & - & - \\ -27 . \quad- & - & 38,0 & - & 39,1 & 96 & 24 \\ -28 . \text { - } & - & 37,8 & - & - & - & - \\ \text { - 1. März } & - & 38,4 & - & - & 100 & 24 \\ \text { - 2. - } & - & 37,3 & - & 38,6 & 88 & 22 \\ - & 3 . \quad- & - & 37,9 & - & - & 96 & 22 .\end{array}$

Am 8. März wurden die Nerven und Muskeln untersucbt und Folgendes gefunden:

rechts

Muse. tib. antic.: galv. $7 \frac{1}{4}$ Milliampères, farad. 36, Contraction langsam.

Nerv. peron.: $\quad-<8 \quad-\quad-\quad$ nicht reizbar

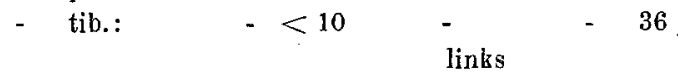

Musc. tib. antic.: galv. 6 Milliampères, farad. 32

Nerv. peron.: $\quad-<8 \quad-\quad-15$

- tib.: $\quad-<8 \quad-\quad-24$.

Es waren demnach nach der Fieberzeit sebr deutliche Störungen in der elektrischen Reizbarkeit der betreffenden Nerven und Muskeln vorhanden. Pat. klagte über beftige Schmerzen und Formication in beiden unteren Extremitäten und Armen. Schmerzhaftigkeit der Nerven und Muskeln der unteren und oberen Extremitäten auf Druck äusserst heftig, Händedruck aufgehoben, kann seine Finger nur mit grosser Mühe flectiren, irgend welcher Druck kanu nicht ausgeübt werden. Mit Ausnabme des Temperatursinnes, der an einzelnen Stellen der Füsse herabgesetzt ist, sind alle Empfindungsqualitäten normal. Schlaf gestört, Appetit gut; alle Bewegungen der Füsse und Unterschenkel herabgesetzt. Patellar- und Achillessehnenreflexe aufgehoben. Bis zum 31. Närz waren Puls, Athmung und Temperatur normal; plötzlich wurde der Puls beschleunigt ohne Temperaturerhöhung. Dies wiederbolte sich am 8. und 9. April. Am 21. März wurde Folgendes festgestellt:

$$
\text { rechts }
$$

Musc. tib. antic.: galv. 6 Milliampères, farad. 51

Nerv. peron.: $\quad-6 \frac{1}{2} \quad-\quad-45$

- tib.: $\quad-\quad 8 \quad-\quad-50$

links

Musc. tib. antic.: galv. $6 \frac{1}{4}$ Milliampères, farad. 35

Nerv. peron.: $\quad-\quad<7 \quad-\quad-40$

- tib.: $\quad-<7 \quad-\quad-38$.

In der fieberfreien Zeit vom 8.-21. März waren demnach die Erscheinungen zurückgegangen. Der Zustand des Kranken besserte sich mehr und mehr, bis er geheilt entlassen wurde. Am 18. Februar ụntersuchte ich sein Milzblut und fand in 3 Präparaten je eine endoglobuläre, ausgewachsene, pigmentirte Amöbe, - der blasse Rand der Blutkörperchen war noch deutlich zu sehen, - ferner einen kleinen, $\frac{1}{4}$ der Grösse eines rothen Blutkörperchens gleich- 
kommenden, sehr beweglichen, mit deutlich beweglichen Pigmentkörnern versehenen, weisslichen Parasiten und endlich ein pigmentirtes, sicbelförmiges Gebilde. Der Kranke litt demnach an einer Malariaerkrankung und waren die Fieber wohl obne Zweifel auf die Entwickelung dieses Parasiten zurückzufübren.

\section{Fall 4.}

Gefangener Sp., junger, kräftig gebauter Javaner, giebt bei seiner Aufnahme an, dass er seit 3 Tagen an Müdigkeit in beiden unteren Extremitäten litte, ist hier und da kurzathmig gewesen. Gesicht ödematös, ebenso besteht Oedem an beiden Unterschenkeln, im Urin kein Eiweiss, Drucksehmerz an den Nervenstämmen und Muskeln der unteren Extremitäten. Einige Tage nach seiner Aufnahme, am 10. Januar 1894, bekam Patient Fieber, Temp. 40,2, während des Fiebers Zunabme der Schmerzen in den unteren Extremitäten, sowie der Kurzathmigkeit. Bis zum 16. war er fieberfrei, Puls und Athmung normal; an diesem Tage wurde der Puls plötzlich beschleunigt, dabei war die Temperatur normal, $36,4^{\circ} \mathrm{C}$. Am 17. Januar Morgens $39,2^{\circ} \mathrm{C}$, Puls 100, Athmung 22 pro Min., Abends $39^{\circ} \mathrm{C}$; am 18. Januar Morgens $38,9^{\circ} \mathrm{C}$, A Abends $39,1^{\circ} \mathrm{C}$; am 19. Januar Morgens $37,3^{\circ} \mathrm{C}$., Abends $39,3^{\circ} \mathrm{C}$. Bis zum 22. Januar bestand unregelmässiges Fieber, zwischen $38,1^{\circ} \mathrm{C}$. und $39,2^{\circ}$ C.; der Puls blieb bei normaler Temperatur bis zum 27. Januar beschleunigt.

Am 19. Januar wurde Pat. elektrisch untersucht:

$$
\text { rechts }
$$

Musc. tib. antic.: galv. 14 Milliampères, farad. 33

Nerv. peron.: $\quad-\quad 2$

- tib.: $\quad-10$

links

Musc. tib.antic.: galv. 4 Milliampères, farad. 43

Nerv. peron.: $\quad->1 \frac{1}{2} \quad-\quad-40$

- tib.: $\quad-\quad 5 \quad-\quad \begin{array}{lll} & - & -\end{array} 5$.

Die Werthe für den faradischen Strom am rechten Nervus peroneus und tibialis konnten wegen enormer cutaner Hyperästhesie nicht bestimmt werden. Am 23. Januar, am Ende der Fiebertage, waren die elektrischen Abweichungen erheblich höher:

rechts

Musc. tib. antic.: galv. < 14 Milliampères, farad. nicht reizbar mit dem

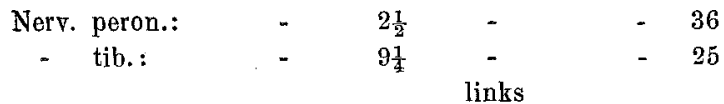
stärksten Strom,

Musc. tib. antic.: galv. 5 Milliampères, farad. 21

Nerv. peron.: $\quad-\quad-2 \frac{1}{2} \quad-\quad-32$

- tib.: $\quad-3 \frac{1}{2}-32$ -

Patient hat heftige Schmerzen am ganzen Körper, intensive Schmerzhaftigkeit auf Druck an den Nerven und Muskeln der unteren Extremitäten, 
Oedem der Unterscbenkel und Füsse, Gang sehr steif, Bewegung der Füsse und Onterschenkel mühsam, Patellar- und Achillessehnenreflexe aufgehoben. Anästhesie am rechten Fussrücken und an der vorderen Oberschenkelfäcbe beiderseits. Herz nach rechts vergrössert; Dämpfung bis zur Mitte des Sternum. Bis zum 6. März blieb Patient fieberfrei; an diesem Tage ein Fieberanfall von $39,6^{\circ} \mathrm{C}$, Puls 104, Athmung 28 pro Min.; am 18. März kebrte das Fieber zum letzten Male zurück, Temp. $39,5^{\circ} \mathrm{C}$., am 19. März $38,6^{\circ} \mathrm{O}$. Von da blieb er fieberfrei. Am 5. April waren die Werthe für die elektrische Reizbarkeit der früher untersuchten Nerven und Muskeln in normale Grenzen zurückgekehrt:

$$
\text { rechts }
$$

Musc. tib. antic.: galv. $7 \frac{1}{2}$ Milliampères, farad. 39

Nerv. peron.: $\quad-4 \quad 4 \quad-\quad 44$

- tib.: $\quad-5 \quad-5 \quad-45$

links

Musc. tib. antic.: galv. $5 \frac{1}{2}$ Milliampères, farad. 39

Nerv. peron.: $\quad-3 \frac{1}{4} \quad-\quad-33$

- tib.: - $61 \frac{1}{4}-36$.

Patient fühlt weder Schmerzen noch Formication, er hat nur noch ein Gefühl von Steif heit in der rechten Kniekehle, Schlaf und Appetit gat, Händedruck kräftig, Drucksehmerz nur gering in den Oberschenkelmuskeln beiderseits. Empfindungsqualitäten überall erhalten. Bewegungen der Füsse und Unterschenkel frei, mit Ausnahme der Dorsalflexion des rechten Fusses, welche noch etwas schwerfällig ausgeführt wird; er läuft noch etwas steif. Patellar- und Achillessehnenreflexe nicht vorhanden. Am 2. Mai war, mit Ausnahme des linken Patellarreflexes, der noch fehlte, der Zustand normal. Patient wurde mit Chinin bebandelt und geheilt entlassen. Am 12. Januar, 2 Tage nach dem ersten Fieberanfall, wurde sein Fingerblut untersucht und in 3 Präparaten 2 endoglobuläre pigmentirte Amöben gefunden, welche die Grösse der Bälfte eines rothen Blutkörpers besassen. Am 23. März und 23. April wurde sein Milzblut mit negativem Resultat auf Malariaamöben untersucht.

Bisweilen stösst man auf Fälle, bei denen die Anamnese und eine noch vorhandene Milzschwellung mit einem hohen Grade von Wahrscheinlichkeit auf eine abgelaufene Malariaerkrankung schliessen lassen, welche man in causalen Zusammenhang mit der multiplen Neuritis zu bringen sich genöthigt sieht. Leyden hat bereits im Jahre 1875 in seiner Klinik der Rückenmarkskrankheiten auf die Lähmungen nach versehiedenen Infectionskrankheiten aufmerksam gemacht; in seinem Vortrage "Ueber die Entzündung der peripherischen Nerven" berührt er diesen Punkt noch einmal. Er sagt auf S. 106 der deutschen Militärärztlichen Zeitschrift, 1888, Heft 3: „Wodurch sollen wir 
uns die Entstehung der multiplen Neuritiden nach acuten Infectionskrankheiten bedingt denken? Sollen wir annehmen, dass die patbogenen Mikroben sich auch in den Nerven, bezw. in deren Scheiden localisiren und hier zu Krankheitserscheinungen Anlass geben? Dies ist für die Lähmungen nach acuten Krankheiten sehr unwahrscheinlich, abgesehen davon, dass niemals der Nachweis von pathogenen Bakterien in den neuritischen Heerden gelungen ist. - Viel wahrscheinlicher ist die Annahme, dass gewisse, durch die Infectionskrankheit gebildete, giftige Stoffe (Ptomaine), wie solche überhaupt einen Theil der Krankheitssymptome hervorrufen, auch die Ursache der multiplen Neuritis sind. - Wir dürfen uns den Vorgang derartig vorstellen, dass sich die toxische Substanz mit einer Substanz der peripherischen Nerven verbindet und dadurch die trophische Degeneration oder den entzündlichen Reiz setzt." Seit dem Vortrage des genannten Autors, der bekanntlich das klinische Bild der multiplen Neuritis zuerst aufgestellt hat, ist eine ganze Reihe von Beobachtungen bekannt geworden, wo nach verschiedenen Infectionskrankheiten die Erscheinungen der multiplen Neuritis auftraten. Wenn man diesen zeitlichen Abstand zwischen der Entwickelung des Krankheitserregers und den darauf folgenden Störungen an den peripherischen Nerven nicht aus dem Auge verliert, wird man in Gegenden, wo die BeriBerikrankheit herrscht, einen Theil der multiplen Neuritiden, die hier ja allgemein unter die Beri-Berierkrankungen gerechnet werden, mit einer überstandenen Malariaerkrankung in $\mathrm{Zu}$ sammenhang bringen können.

Ich möchte auch hierfür kurz zwei Beispiele anführen:

\section{Fall 1.}

Der Gefangene T. wurde am 7. Mai 1894 in's Krankenbaus aufgenommen. Er hatte seit 12 Tagen Schmerzen, sowie ein Gefühl von Ameisenlaufen und Müdigkeit in den unteren und oberen Extremitäten, seit 3 Tagen litt er an Oedem des Gesichtes und der Unterschenkel. In den ersten Tagen der Erkrankung batte er Fieber, und zwar begann dasselbe am Abend, den Tag über war er fieberfrei und arbeitete dann in den ersten Tagen der Erkrankung; wäbrend des Fiebers nahmen die Sehmerzen zu. Appetit ist bei seiner Aufnabme schlecht, Schlaf gut, Blasen- und Darmfunction zeigen keine Abweichungen, eben so wenig die verschiedenen Empfindungsqualitäten. Mrskeln und Nerven der oberen und unteren Extremitäten auf Druck schmerz- 
haft. Patellar- und linker Achillessehnenreflex erhalten, der rechte nicht vorbanden. Bewegung der Füsse und Untersebenkel schwerfälig, ebenso die der Hände; Händedruck herabgesetzt. Herz etwas nach rechts vergrössert. Kein Eiweiss im Urin. Eine Milzschwellung war nicht mebr zu constatiren. Die etwas spät vorgenommene elektrische Untersuchung der Nerven und des Musc. tib. antic. beider Unterschenkel ergab am 30. Mai eine Abnahme der faradischen Reizbarkeit des rechten Musc. tib. antic. Patient hatte während der Zeit seiner Bebandlung niemals schnellen Puls, Athmung oder erhöhte Temperatur. Am 13. Juni wurde er geheilt entlassen, Druckschmerz verschwonden, Händedruck kräftig, Bewegungen der Extremitäten frei, der rechte Acbillessehnenreflex vorhanden. Die elektrische Reizbarkeit auf den faradischen Strom war am Musc. tib. antic. dext. in normale Grenzen zurückgekebrt. Die Untersuchung des Blutes auf Plasmodien ergab negative Resultate.

\section{Fall 2.}

Seit 2 Wochen hatte der Gefangene P. Schmerzen in den Kniegelenken und Formication, sowie ein Gefähl von Wärme in den Waden. Vor dem Eintritt dieser Erscheinungen hatte er 2 Monate anbaltendes Fieber gehabt. Die Milz war bei der Aufnahme vergrössert, Ort- und Tastsinn waren an beiden Unterschenkeln aufgehoben; die anderen Empfindungsqualitäten normal, Druckschmerz an den Nerven und Muskeln der Unterschenkel vorhanden. Patellar- und Achillessehnenreflex feblen, am Herzen wurde nichts Bemerkenswerthes gefunden. Die Erregbarkeit auf den faradischen Strom war am rechten Nerv. tib. und linken Nerv. peron. herabgesetzt; Puls, Athmung, Temperatur normal, sie blieben es bis zu seiner Entlassung. Einen Monat nach seiner Aufnabme war der Zustand besser, die Schmerzhaftigkeit auf Druck war verschwunden, Ort- und Tastsinn vorbanden. Nach einem weiteren Monat waren ausser dem Feblen der Patellarund Achillessehnenreflexe alle Krankbeitserscheinungen geschwunden. Auch bei diesem Kranken wurden niemals Plasmodien gefunden.

Dass die Malaria einen gewissen Antheil hat an der Entstehung und Entwickelung der multiplen Neuritiden, die man in tropischen Gegenden oder Ländern, wo die Beri-Berikrankheit herrscht, als Beri-Berierkrankungen bezeichnet, geht auch aus epidemiologischen Beobachtungen hervor. Auf Plätzen im malaiischen Archipel, wie Poelve Bras und dem früheren Onrust, wo Malaria in besonderer Häufigkeit und Heftigkeit, zugleich mit Beri-Berierkrankungen sich zeigte, zeichnen sich die letzteren gegenüber anderen, weniger von Malaria heimgesuchten Plätzen durch Ausbreitung und Intensität aus. Scheube gedenkt in seinem letzten Werk "Die Beri-Berikrankheit" der 3 Hauptheerde der Beri-Beri, nehmlich der brasilianischen Hochebenen, 
Niederländisch-Indien und Japan. Die höchste Mortalitätsziffer liefert Brasilien, ein Land, welches ausgesprochene Malariaheerde besitzt; ihm zunächst steht Niederländisch-Indien, wo wir ebenfalls die Malariaerkrankungen in grosser Ausbreitung vorfinden; am geringsten ist die Mortalität an Beri-Beri in Japan, wo, wie uns Hirsch in seinem bekannten Werk mittheilt, die Malariaerkrankungen viel geringer sind und in milderen Formen verlaufen. Ich erkläre mir den günstigen Einfluss, welchen die Entfernung eines Beri-Berikranken aus dem Ort der Infection nach Beri-Beri-freien Gegenden ausübt, nun auch theilweise damit, dass in diesen auch meist Malaria-freien Gebirgsplätzen ausser der Verhütung der Aufnahme eines noch später zu beschreibenden und multiple Neuritis hervorbringenden, amöboiden Organismus die Complication einer Malariainfection verhindert wird, die, wie ich an einer ganzen Reihe von Fällen im hiesigen Krankenhause beobachten konnte, das lethale Ende hervorrief oder beschleunigte. Wahrscheinlich haben den älteren Autoren, die über Beri-Beri geschrieben haben, ähnliche Fälle, wie die oben mitgetheilten, vorgeschwebt, wenn sie, wie $\mathrm{Neeb}$, Bauer, Anderson und Andere, die Beri-Beri für eine Art von Malaria erklärten. Ausser den multiplen Neuritiden, bei denen die Plasmodien der Malaria als Ursache verantwortlich gemacht werden mussten, giebt es nun eine weitere grosse Reihe von Fällen, die, wie ich hier besonders hervorheben will, den von mir in diesem Archiv beschriebenen, intermittirenden Gang der Pulscurve erkennen lassen, bei denen man keine Malariaplasmodien, dagegen einen anderen amöboiden Organismus findet, der nach meinen bisherigen Untersuchungen morphologisch von den Malariaplasmodien getrennt, und weil er in der grössten Zahl der Fälle vorhanden ist, mit sebr viel Wahrscheinlichkeit als die Ursache dieser Reihe von multiplen Neuritiden angesehen werden muss. Ich werde über diesen Mikroorganismus, der meines Wissens noch nicht beschrieben wurde, später berichten.

Der Zweck dieser Mittheilung ist, die Auffassung der meisten Autoren, als sei die Ursache der Beri-Beri eine einheitliche, zu widerlegen. 